\title{
High-fidelity simulation increases obstetric self-assurance and skills in undergraduate medical students*
}

\author{
Christoph Scholz ${ }^{1, \mathrm{a}, * *}$, Corinna Mann ${ }^{2, \mathrm{a}}$, Veronika \\ Kopp $^{3}$, Bernd Kost ${ }^{2}$, Franz Kainer ${ }^{2}$ and \\ Martin R. Fischer ${ }^{3}$ \\ ${ }^{1}$ Department of Obstetrics and Gynaecology, Ulm \\ University, Ulm, Germany \\ ${ }^{2}$ Department of Obstetrics and Gynaecology, Ludwig- \\ Maximilians-University, Munich, Germany \\ ${ }^{3}$ Department of Teaching and Educational Research in \\ Medicine, Ludwig-Maximilians-University, Munich, \\ Germany
}

\begin{abstract}
Objective: Teaching intrapartum care is one of the most challenging tasks in undergraduate medical education. Highfidelity obstetric simulators might support students' learning experience. The specific educational impact of these simulators compared with traditional methods of model-based obstetric teaching has not yet been determined.

Study design: We randomly assigned 46 undergraduate medical students to be taught using either a high-fidelity simulator or a scale wood-and-leather phantom. Their self-assessments were evaluated using a validated questionnaire. We assessed obstetric skills and asked students to solve obstetric paper cases.

Main outcome measures: Assessment of fidelity-specific teaching impact on procedural knowledge, motivation, and interest in obstetrics as well as obstetric skills using high- and low-fidelity training models.

Results: High-fidelity simulation specifically improved students' feeling that they understood both the physiology of parturition and the obstetric procedures. Students in the simulation group also felt better prepared for obstetric house jobs and performed better in obstetric skills evaluations. However, the two groups made equivalent obstetric decisions.
\end{abstract}

\footnotetext{
*Funding was provided by the Committee on the Allocation Students Tuition Fees for purchasing the simulator. The study itself was funded by the Faculty of Medicine.

${ }^{a}$ These two authors contributed equally.

***Corresponding author:

PD Dr. Christoph Scholz

Department of Obstetrics and Gynaecology

Ulm University

Prittwitzstrasse 43

89075 Ulm

Germany

E-mail: christoph.scholz@uniklinik-ulm.de
}

Conclusion: This study provides first data on the impact of high-fidelity simulation in an undergraduate setting.

Keywords: Clinical competence; obstetric simulation; teaching methods; undergraduate medical education.

\section{Introduction}

For pregnant women, giving birth is the culmination of the reproductive process. For most medical students, experiencing the delivery of a baby constitutes the culmination of their obstetric clerkship. Integrating medical students into intrapartum care, however, is far from easy. Many factors contribute to this challenge: for parturients, childbirth has increasingly become a once-in-a-lifetime event and medical students tend not to be part of the stage plan. The frequency of litigation has labeled obstetrics as a high-risk endeavor in which anything not contributing to safety, but rather distracting from the primary task of ensuring the well-being of mother and child is seen as intolerable [6]. As a result, being involved in intrapartum care is a difficult task for medical students, especially for male medical students [7], and such daunting experiences can discourage medical students from further engaging in obstetrics and lead them to choose a different career option. It is a constant challenge to recruit quality students into obstetrics and gynecology and any help to infuse students with enthusiasm for our speciality should be welcomed [3].

It is well known that undergraduate experiences have a high impact on future career choices and medical schools consider undergraduate education in obstetrics and gynecology to be a very important focal point. The 2002 reform physician licensing legislature has further increased the weight placed on undergraduate obstetrics and gynecology training in Germany [16]. One way to experience practical aspects of obstetric cases is through simulator-based learning. Visualization of obstetric relations using fetal and pelvic models is a timehonored way of teaching cardinal movements of labor and may also be accompanied by case-based scenarios. This kind of low-fidelity model has been a part of our undergraduate obstetrics and gynecology course for many years. LudwigMaximilians-University of Munich has recently introduced high-fidelity simulator training into undergraduate obstetric teaching. A mock-delivery suite has been integrated into a newly established skills laboratory equipped with a wireless high-fidelity obstetric simulator that displays maternal vital signs, fetal descent, and CTG tracings and is able to dynamically integrate these parameters into case scenarios. To date, high-fidelity obstetric simulation has been mainly integrated 
into postgraduate obstetric education, and its success in an undergraduate course is as yet largely unknown.

To our knowledge, the only published study so far that compared high-fidelity obstetric simulation against no training at all found an increased student self-rated confidence toward obstetric procedures after high-fidelity simulator training compared to students who received no training at all [12]. However, the specific impact of a high-tech, high-cost environment on medical students' learning experience and acquisition of obstetric skills has not yet been investigated.

In this study, we compared undergraduate students' selfassessment and overall performance before and after the obstetrics course, with special attention to gender differences. Second, we specifically looked for differences between students trained with low- vs. high-fidelity obstetric teaching methods by comparing their self-assessment data and their performance improvement, again looking for possible gender differences.

\section{Methods}

\section{Sample, study design, and procedure}

A sample of 63 medical students (42 women and 21 men, semesters 8-10) was assigned to a 5-week obstetric course in the Department of Obstetrics and Gynaecology of the Ludwig-Maximilians-University in Munich (Innenstadt Campus). Sample size was determined by the clinical rotation scheme set by the medical faculty. Two groups of 23 students (18 women and 5 men) were randomly sampled. In a second step, we controlled for comparability of the two groups concerning gender, age, prior knowledge, and previous obstetric teaching. One group participated in traditional obstetric training on a scale wood-and-leather phantom and the other in a high-fidelity training course.

Parallelization was successful because of the homogeneity of the given sample of medical students. Differences in age between the groups of high-fidelity (mean $\pm \mathrm{SD}, 26.17 \pm 3.27$ ) and low-fidelity (mean $\pm \mathrm{SD}, 26.17 \pm 3.27$ ) training are statistically not significant $[t(44)=0.64, \mathrm{P}=0.528]$. Self-assessment of prior knowledge on a 6-point Likert scale ranging from 1 ("I completely disagree") to 6 ("I completely agree") led to the same extent in the high-fidelity $($ mean $\pm S D, 1.78 \pm 1.11)$ and low-fidelity (mean $\pm S D, 1.89 \pm 1.02)$ ( $\mathrm{Z}=-0.35, \mathrm{P}=0.724)$ groups.

All students were assessed for motivation and capacity for clinical obstetric judgment at the end of their formal obstetric instruction but before practical experience on the labor ward. Formal obstetric instruction included a lecture on labor and the conduct of delivery followed by either high-fidelity interactive obstetric simulation $(n=23)$ or traditional obstetric teaching $(n=23)$ using a clinical scenario with a paper-strip CTG tracing and a scale wood-and-leather birth model to visualize fetal station and lie. After the study, all students had the opportunity to take part in a high-fidelity teaching session on a voluntary basis.

\section{Characteristics of the obstetric models}

The scale wood-and-leather phantom (Figure 1, lower row) has been used for many years to teach the cardinal movements of labor to undergraduate students. This phantom is integrated into a standardized method of presenting obstetric cases. A leather baby is manually pushed through the pelvis while an intrapartum scenario is described, which is accompanied by a paper-strip CTG tracing.

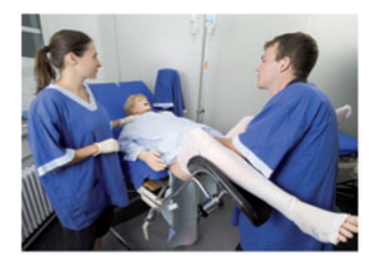

- Interactive full-sized anthropomorphic robotic birth simulator

- Online, dynamic maternofetal parameters and clinical scenario

- High-cost
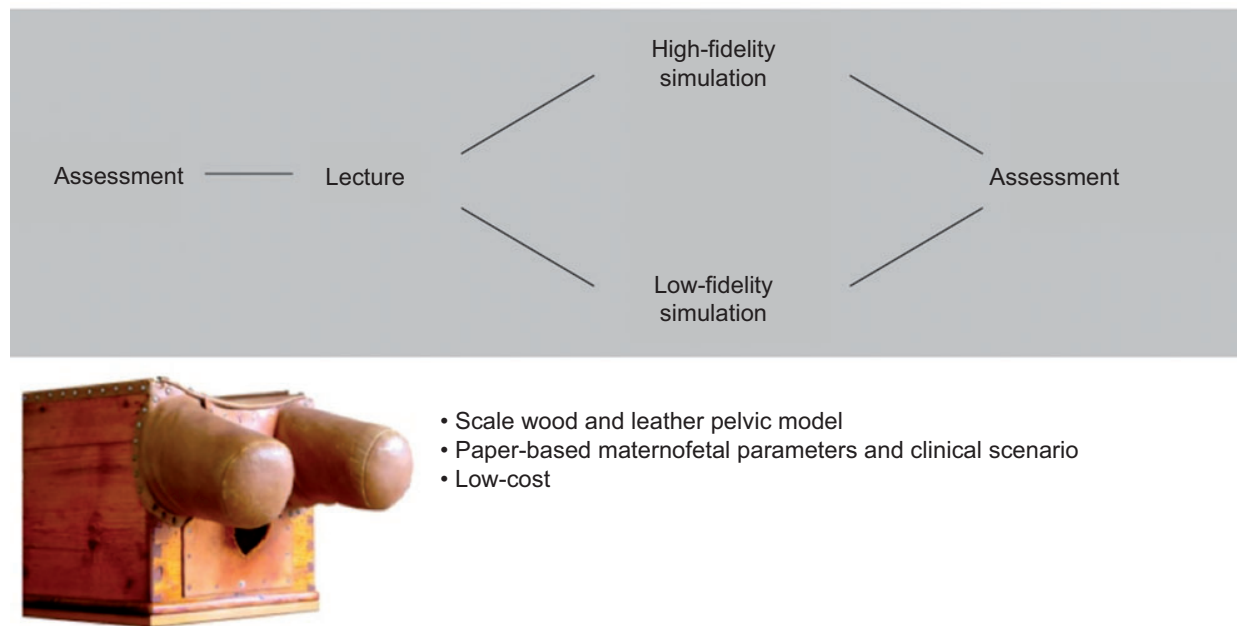

- Scale wood and leather pelvic model

- Paper-based maternofetal parameters and clinical scenario

- Low-cost

Figure 1 Study design and model characteristics.

Flowchart of the study design is depicted in the middle. Both simulators used in this study are shown as well as their most important characteristics. 
Table 1 Descriptive statistics: results (mean \pm SD, minimum, and maximum) from self-assessment and performance of the medical students in the two groups, high-fidelity $(\mathrm{n}=23)$ and low-fidelity training $(\mathrm{n}=23)$, are shown.

\begin{tabular}{|c|c|c|c|c|}
\hline & \multicolumn{2}{|c|}{ High-fidelity training $(\mathrm{n}=23)$} & \multicolumn{2}{|c|}{ Low-fidelity training $(\mathrm{n}=23)$} \\
\hline & Mean \pm SD & Min-max & Mean \pm SD & Min-max \\
\hline \multicolumn{5}{|l|}{ Self-assessment scales } \\
\hline Comprehension/preparation & $4.07 \pm 0.74$ & $2.83-5.50$ & $3.54 \pm 0.79$ & $2.17-5.17$ \\
\hline Procedural knowledge & $3.76 \pm 0.76$ & $2.60-5.00$ & $2.83 \pm 1.13$ & $1.00-5.20$ \\
\hline Motivation & $4.14 \pm 0.63$ & $3.17-5.33$ & $4.06 \pm 1.06$ & $2.17-5.83$ \\
\hline Expectation & $5.10 \pm 0.55$ & $4.25-6.00$ & $5.11 \pm 0.77$ & $3.25-6.00$ \\
\hline Interest & $4.40 \pm 0.94$ & $2.50-6.00$ & $4.92 \pm 1.13$ & $2.50-6.00$ \\
\hline \multicolumn{5}{|l|}{ Performance tests } \\
\hline Obstetric skills & $6.18 \pm 1.56$ & $3.00-8.00$ & $3.96 \pm 2.29$ & $1.00-9.00$ \\
\hline CTG tracing & $17.70 \pm 4.44$ & $3.00-22.00$ & $12.74 \pm 4.92$ & $0.00-21.00$ \\
\hline Obstetric decision-making & $2.35 \pm 1.07$ & $0.00-4.00$ & $1.74 \pm 1.54$ & $0.00-5.00$ \\
\hline
\end{tabular}

Medical students are thus taught obstetric management by integrating maternofetal data with pelvic examination results.

Noelle S575 (Gaumard Scientific, Coral Gables, FL, USA) is an interactive full-sized female anthropomorphic robotic birth simulator (Figure 1, upper row). Apart from being a full-scale cardiac life-support mannequin with physical representation of heartbeat, breathing, and blood pressure, it is also equipped with a mechanical birthing system. Online monitor tracings are available for maternal vital signs and CTG. The mannequin is controlled wirelessly and can display prearranged clinical scenarios. Noelle S575 is now integrated into a designated obstetric teaching room in the skills laboratory of the medical school.

\section{Instruments}

Self-assessment questionnaire A paper-and-pencil questionnaire for the self-assessment of students in an obstetric training was developed. The questionnaire consisted of 29 items in five scales (Table 2). All questions were answered on a 6-point Likert scale ranging from strongly disagree to strongly agree (1="I completely disagree", 2="I disagree", 3="I rather disagree", 4="I rather agree", 5="I agree", 6="I completely agree"). The self-assessment tool was constructed for the purpose of the study and reviewed by independent experts for face validity. Reliability scores of scales are reported. No prior piloting was done. Reliability analyses were performed to determine the internal consistency of the self-assessment questionnaire. Further validation for the refinement of the questionnaire is needed and will be done in further studies. The results are indicated as Cronbach $\alpha$ for each of the five scales.

- "Comprehension": Self-assessment concerning the comprehension of obstetric parameters of six items (e.g., "I understand the course of events of a physiological delivery."). The scale reached a reliability of $\alpha=0.73$ (Cronbach $\alpha$ ). We considered Cronbach $\alpha$ of 0.80 as the gold standard of reliability.

- "Procedural knowledge": Self-assessment on this scale concerning the procedures of intrapartum care was composed of five items (e.g., "I feel confident delivering the fetal head."). Cronbach $\alpha$ was $\alpha=0.89$.

- "Motivation": Motivation of students during the training to learn in an obstetric environment was assessed using six items (e.g., "Learning gives pleasure to me."). Cronbach $\alpha$ was $\alpha=0.74$.

- "Expectation": On this scale, the expectation of learning basics of intrapartum care was assessed using four items (e.g., "The medical care during labor is too complicated for me."). Cronbach $\alpha$ was $\alpha=0.53$.
- "Interest": Interest in obstetrics was measured on a scale consisting of six items (e.g., "I am interested in the topic intrapartum care."). Cronbach $\alpha$ was $\alpha=0.91$.

In summary, a good reliability of the self-assessment questionnaire with 29 items in five scales can be accepted as given. Four out of five scales reached an adequate reliability level (Cronbach $\alpha>0.70$ ) and two scales reached a high reliability level (Cronbach $\alpha \sim 0.90$ ) (Table 2).

Performance tests Performance of the students was operationalized using three tests, which focused on different requirements of professional work in an obstetric ward.

- "Obstetric skills": The first tested obstetric skills and consisted of six tasks. Students had to designate partogram parameters and assign maternal as well as fetal structures that could be palpated by a vaginal examination. The maximum score was 10 points. The test had a reliability of $\alpha=0.58$ (Cronbach $\alpha)$.

- "CTG tracing": In a second test, participants had to interpret CTG tracings according to the FIGO criteria [15, 17]. Fetal heart rate tracings had to be graded as "normal," "suspicious," or "pathologic" and were chosen to unambiguously fall into one of these categories (Figure 2). Three independent experts verified the respective classifications. The maximum score was 24 points. The test's reliability was adequate with $\alpha=0.70$ (Cronbach $\alpha$ ).

- "Obstetric decision-making": The third performance test was on obstetric decision-making and consisted of six short clinical case scenarios, in which medical history, CTG tracing, maternal vital signs as well as pelvic examination results had to be integrated into an obstetric decision regarding further management. Students had to choose one best answer out of five alternatives. A maximum of six points could be achieved. Cronbach $\alpha$ was $\alpha=0.72$.

In summary, the results of reliability analyses offered an adequate internal consistency of performance testing with two out of three scales reaching a reliability level of Cronbach $\alpha>0.70$.

\section{Data and statistics}

Descriptive statistics (mean $\pm \mathrm{SD}$, minimum, and maximum) of self-assessment and performance of the medical students are listed (Tabel 1). Differences in central tendency between the two groups (high- vs. low-fidelity obstetric training) were tested for significance by Mann-Whitney $U$-test for independent samples ( $U$-score). 
Table 2 Questions on the self-assessment questionnaire: translation of the wording of the self-assessment questions.

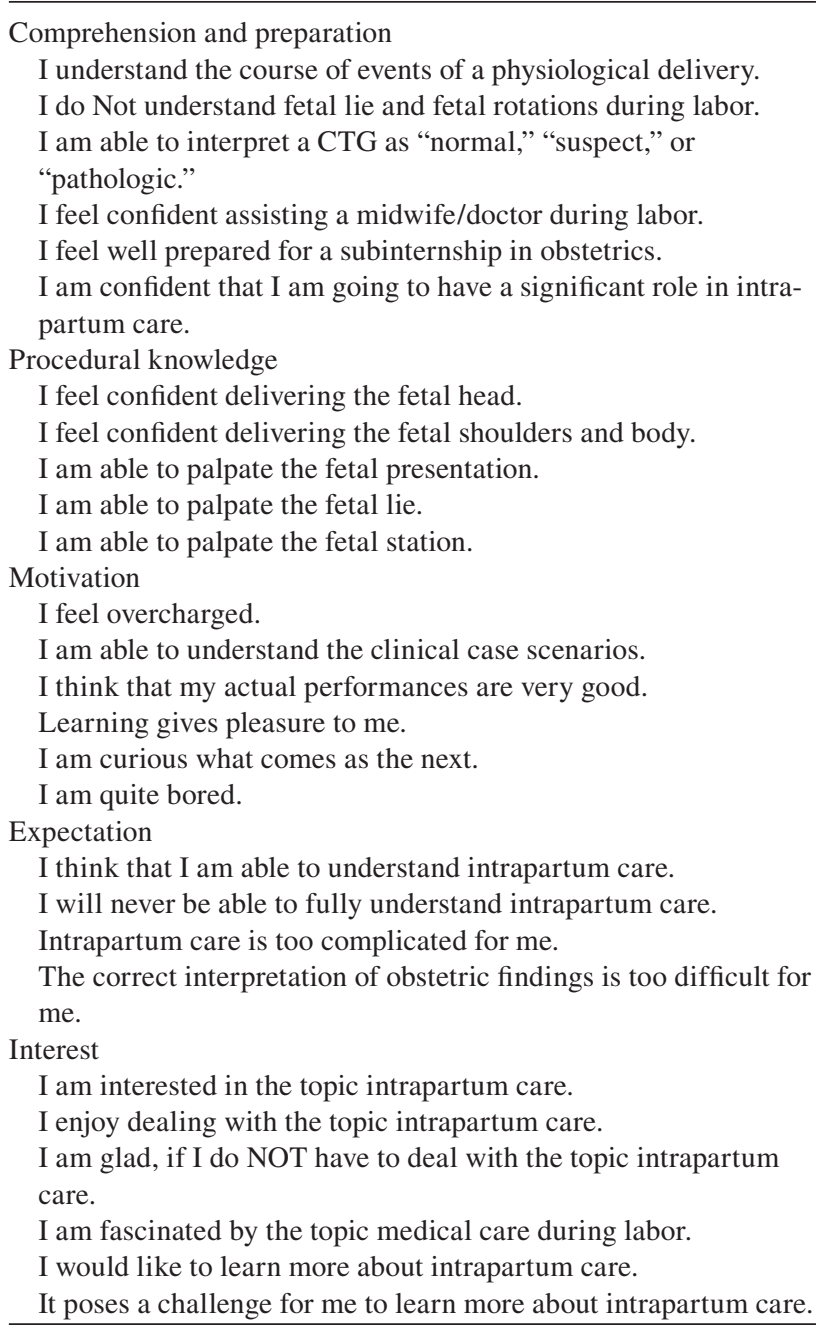

Possible effects of age, gender, and prior knowledge on the outcome were examined with correlation analyses (Spearman $\rho$ ). Data were analyzed using the Statistical Package for the Social Sciences software version 17.0 (SPSS Inc., Chicago, IL, USA). An $\alpha$-level of 0.05 was used for all statistical analyses. To compare the means, we used the Student $t$-test. For effect size, Cohen $d$ was calculated [2].

\section{Results}

\section{Comparison of students' self-assessment using low- and high-fidelity models}

Significant differences between the two groups were revealed in two out of five scales of the self-assessment questionnaire, both with a higher score of the high-fidelity group (Figure 3).

Compared with traditional model-based teaching, highfidelity simulation significantly improved students' selfassessment regarding their comprehension of the physiology of vaginal delivery and their self-assessed preparedness to engage further in obstetrics. In this scale, low- and highfidelity-trained students differed significantly ( $U=166$, $\mathrm{P}=0.031)$. The effect size was of medium practical relevance $(d=0.70)$.

Regarding obstetric procedures (procedural knowledge), high-fidelity-trained students felt significantly more confident in delivering the baby and palpating fetal structures during delivery than low-fidelity-trained students $(U=128, \mathrm{P}=0.004)$. This effect was of practical relevance $(d=0.99)$.

Concerning the self-assessment scales motivation $(U=239$, $\mathrm{P}=0.962)$, expectation $(U=232, \mathrm{P}=0.631)$, and interest ( $U=179, \mathrm{P}=0.094$ ), groups did not differ significantly.

All results from students' self-assessments were gender independent, but there were significant effects of age on the scales procedural knowledge $(\mathrm{r}=0.365, \mathrm{P}=0.014)$, motivation $(\mathrm{r}=0.408, \mathrm{P}=0.006)$, and expectation $(\mathrm{r}=0.389, \mathrm{P}=0.008)$. The three significant correlations were of positive proportionality, with a higher self-assessment score of older students. Furthermore, prior knowledge showed positive correlations with the scales comprehension and preparation $(\mathrm{r}=0.384$, $\mathrm{P}=0.008)$ and interest $(\mathrm{r}=0.361, \mathrm{P}=0.015)$.

\section{Comparison of students' performance using low- and high-fidelity models}

The performance of medical students with regard to vaginal examination skills and plotting these results on a partogram depends on the training with significant differences between the two groups with a higher score of the high-fidelity group. This difference between the two groups was significant $(U=105, \mathrm{P}=0.001)$ and of practical relevance $(d=0.97)$ (Figure 4A).

Furthermore, CTG tracings were interpreted significantly better by students who experienced high-fidelity teaching ( $U=104, \mathrm{P}=0.000)$. Their grading of fetal heart rate tracing was more often in the appropriate category. The difference between the groups was of practical relevance $(d=0.94)$ (Figure 4B).

However, when asked to integrate these data within a given clinical scenario and decide on further obstetric management, the groups of low- and high-fidelity simulation produced equivalent results $(U=184, \mathrm{P}=0.072)$ (Figure $4 \mathrm{C}$ ).

All results of performance tests were independent of the matching variables age, gender, and prior knowledge. No significant correlation could be indicated.

\section{Discussion and conclusion}

Obstetric model-based teaching is a time-honored way of instructing those involved in childbirth. Madame du Coudray's "machine," built in the $18^{\text {th }}$ century, is thought to be the first obstetric simulator and closely resembles the scale wood-andleather phantoms used today (Figure 1) [1]. Modern technology has opened greater possibilities of simulating human physiology, which were first used to train advanced cardiac life support (ACLS). Quite recently, obstetric simulators that combine these ACLS simulators with obstetric parameters 
CTG

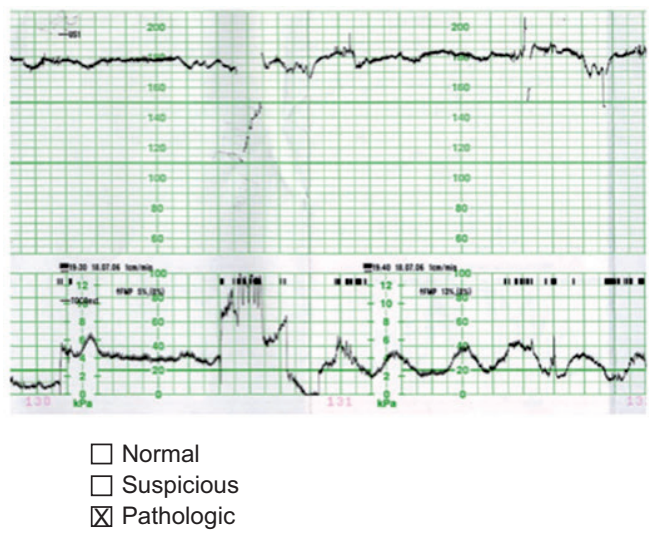

History

28 (1G/0P) 34+5 weeks of gestation. Estimated fetal weight on $10^{\text {th }}$ percentile. "lower UTI" treated with Amocixillin (500 mg, po, tid) since $24 \mathrm{~h}$, contractions

$\begin{array}{lll}\text { Maternal parameters } & \cdot H R: & 115 \mathrm{bpm} \\ & \cdot \mathrm{NIBP} & 105 / 60 \mathrm{mmHg} \\ & \cdot \text { RR: } & 25 \mathrm{bpm} \\ & \cdot \text { Temp.: } & 38.2^{\circ} \mathrm{C} \\ & & \\ & & \\ \text { Pelvic examination } & \cdot \text { Dilatation: } & \text { Early effacement } \\ & \cdot \text { Presentation: } & \text { Vertex; LOA } \\ & \cdot \text { Station: } & -4\end{array}$



$\square$ CT-scan to exclude pulmonary embolism

Figure 2 Example of a paper-based case on which students had to come to a decision regarding further obstetric management.

have become available. The Noelle S575 used in our study is one such state-of-the-art simulator [8].

In this study, we investigated the impact of high-fidelity simulation on students' perception of their obstetric knowledge and dexterity as well as their preparedness to enter into an optional subinternship in obstetrics. Skills in assessing intrapartum parameters, such as fetal heart rate tracings and vaginal examination results were tested. We also requested students to integrate these maternofetal parameters into a given clinical scenario and provide a decision on further obstetric management. In the study population of 46 students, we found that model-based training in conjunction with formal obstetric teaching improved students' overall performance regardless of the type of obstetric phantom used. High-fidelity simulation, however, improved specific aspects of students' confidence in their obstetric abilities. These aspects included the notions that they understood the physiology of parturition and felt better prepared for obstetric house jobs. Furthermore, they felt significantly more knowledgeable regarding the procedures of parturition. Students' self-assessment of their medical skills tends to predict their performance in these specific tasks as well as examination results [18]. Indeed, after

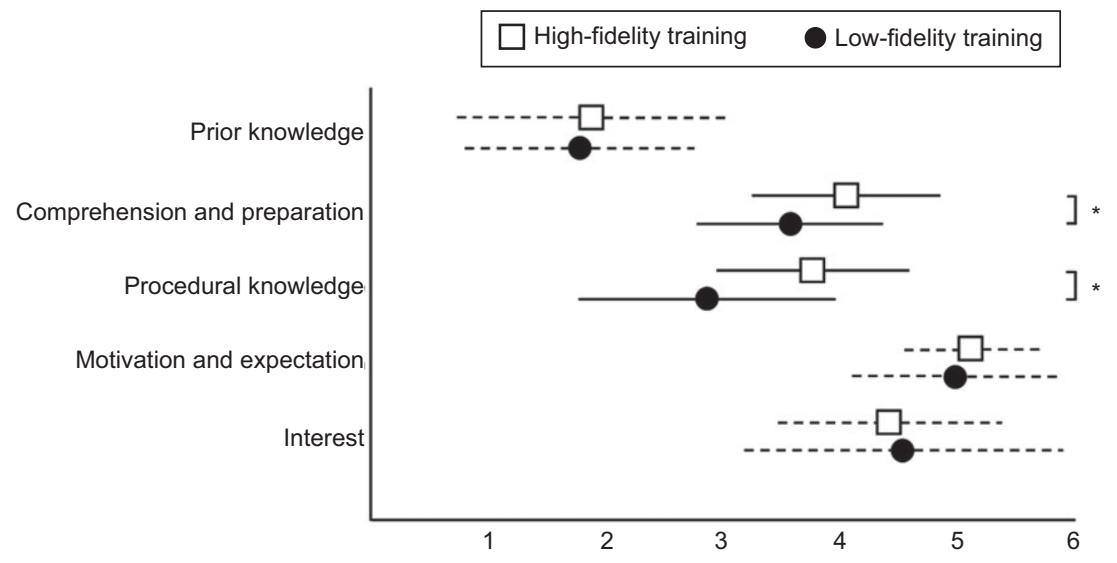

Figure 3 Results of self-assessment questionnaire.

Students had to agree or disagree to specific questions on the indicated scales. Squares indicate students who experienced high-fidelity training; filled circles, students who experienced low-fidelity training. Statistical significance $(\mathrm{P}<0.01)$ is indicated with an asterisk and bracket. Error bars indicate SD. For results without significant differences, error bars are dotted. 
A Obstetric skills



B CTG tracings

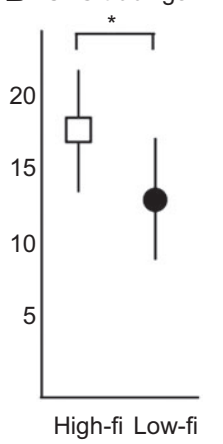

C Obstetric decision-making

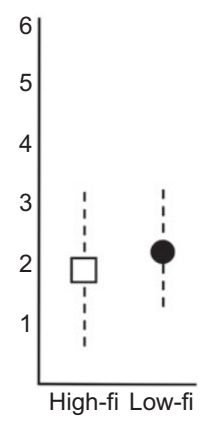

Figure 4 Results of the performance tests.

(A) Students' performance in obstetric skills. This test included designating palpable structures on pelvic examination as well as partogram parameters. (B) Test in which students were faced with decisions on further obstetric management after reading a paperbased case study comprising "history", "CTG tracing", "maternal parameters", and "pelvic examination". Statistical significance $(\mathrm{P}<0.01)$ is indicated with an asterisk and bracket. Error bars indicate SD. "High-fi" and "Low-fi" stand for high-fidelity training or lowfidelity training, respectively.

high-fidelity simulation, students performed better in specific obstetric skills, i.e., interpreting fetal heart rate tracings and designating partogram parameters as well as describing intrapartum pelvic examination results. However, when asked to integrate this information into an obstetric decision on further management, students showed equivalent results after highand low-fidelity simulation.

Jude et al. [12] described the positive impact of highfidelity simulation on measures of confidence in a group of 33 students with high-fidelity training vs. no training. In our study, students' self-assessment and performance improved considerably by means of model-based training regardless of its fidelity. To the best of our knowledge, however, no studies have been published that evaluated the specific impact of a state-of-the-art high-fidelity interactive obstetric simulator as compared with classical obstetric instruction using a static birth model in an undergraduate setting. This classical model has undisputable advantages. It is available at an affordable price, has almost no maintenance cost, and requires little time to become familiar with its features. When this model is combined with case-based scenarios and CTG tracings, students can be trained in the essential skills of intrapartum care. They feel motivated and prepared to engage in obstetrics and generally perform better in obstetric tasks. Therefore, model-based teaching as such has a positive impact on students' obstetric motivation and performance and should be incorporated into an undergraduate course whenever possible.

There are solid data that high-fidelity simulation cannot serve as a substitute for real-life exposure to real clinical challenges [13, 10]. Instead, it should be integrated as an incremental course concept, in which simulation prepares for reality and trains specific aspects that are essential for optimal performance. Medical simulations should be highly relevant and not necessarily highly realistic [11]. It is therefore important to determine what is relevant for undergraduate medical students. A recent review on the APGO Medical Student Objectives in Obstetrics and Gynaecology defined top priority learning objectives for undergraduate medical students [4]. Of these objectives, our study found a specific improvement by means of high-fidelity training in "physical examination skills", "routine antepartum care", and "intrapartum care". Students felt more secure in performing pelvic examinations and were able to communicate results in a well-organized manner in a partogram.

Arguably, most importantly, our study showed that highfidelity simulation specifically increased students' self-esteem in terms of being an integral part of obstetric care. They felt they better understood the physiology of labor and felt more secure in delivering the baby. Both competencies are essential when students need to consider themselves as useful members of obstetric care teams. Furthermore, after high-fidelity simulation, students proved to be more able to correctly interpret fetal heart rate tracings and assess maternofetal parameters. These qualities are likely to make those students valuable junior members of real-life obstetric care and might make their first experiences in delivery suites less disheartening [9]. All of these effects were true for male and female medical students alike.

Integrating the multifaceted data of intrapartum care into an obstetric decision, however, was not increased by high-fidelity simulation compared with traditional ways of demonstrating intrapartum phenomena. These decisions may not be required from medical students, although we still hoped that these case studies would assess students' grasp of obstetric pathophysiology [4]. We argue that students are able to integrate a set amount of information into a clinical decision and that the interdependence of fetal station and lie as well as CTG tracing and maternal parameters might exceed this amount regardless of the fidelity of the model on which they were trained [5, 14]. This, however, might not be true obstetric postgraduate simulator-based training, as this is currently part of strategies to implement risk-management concepts into obstetric care.

Additional studies are needed to evaluate the ability of available technology to support undergraduate teaching. Our data give rise to the hope that high-fidelity simulation integrated into an undergraduate course prepares and motivates students to engage in obstetric care. It might also improve students' obstetric learning experiences, infuse students with enthusiasm for obstetrics, and eventually recruit more male and female medical students into the specialty.

\section{Acknowledgments}

We would like to thank the LMU Students Tuition Fee Committee for funding the high-fidelity simulator and providing training opportunities in the skills laboratory. Furthermore, we would like to thank the many students of the skills laboratory team led by Kirsten Eghardt for providing logistical support for this study.

\section{References}

[1] Berge AFL. The king's midwife: a history and mystery of Madame du Coudray. Med Hist. 2000;44:561-2. 
[2] Cohen J. Statistical power analysis for the behavioral sciences New York, NY: Lawrence Erlbaum; 1988.

[3] Dunn TS, Wolf D, Beuler J, Coddington CC. Increasing recruitment of quality students to obstetrics and gynecology: impact of a structured clerkship. Obstet Gynecol. 2004;103: 339-41.

[4] Erickson SS, Metheny WP, Cox SM, Bienstock JL, Espey EL, Goepfert AR, et al. A comprehensive review to establish priority learning objectives for medical students in the obstetrics and gynecology clerkship. Am J Obstet Gynecol. 2008;199:563. e1-9.

[5] Gerjets P, Scheiter K, Catrambone R. Designing instructional examples to reduce intrinsic cognitive load: molar versus modular presentation of solution procedures. Instruct Sci. 2004;32:33-58.

[6] Grunebaum A. Error reduction and quality assurance in obstetrics. Clin Perinatol. 2007;34:489-502.

[7] Hamilton A. 'GAMMS': go away, male medical student. Stud Br Med J. 2006;14:112-3.

[8] Hammoud MM, Nuthalapaty FS, Goepfert AR, Casey PM, Emmons S, Espey EL, et al. To the point: medical education review of the role of simulators in surgical training. Am J Obstet Gynecol. 2008;199:338-43.

[9] Higham J. How can we make our medical students enthusiastic about a future in obstetrics and gynaecology? Br J Obstet Gynaecol. 2006;113:499-501.

[10] Issenberg SB, McGaghie WC, Petrusa ER, Lee Gordon D, Scalese RJ. Features and uses of high-fidelity medical simulations that lead to effective learning: a BEME systematic review. Med Teach. 2005;27:10-28.
[11] Johannsson H, Ayida G, Sadler C. Faking it? Simulation in the training of obstetricians and gynaecologists. Curr Opin Obstet Gynecol. 2005;17:557-61.

[12] Jude DC, Gilbert GG, Magrane D. Simulation training in the obstetrics and gynecology clerkship. Am J Obstet Gynecol. 2006;195:1489-92.

[13] Macedonia CR, Gherman RB, Satin AJ. Simulation laboratories for training in obstetrics and gynecology. Obstet Gynecol. 2003;102:388-92.

[14] Mayer RE, Moreno R. Nine ways to reduce cognitive load in multimedia learning. Educ Psychol. 2003;38:43-52.

[15] Schiermeier S, Pildner von Steinburg S, Thieme A, Reinhard J, Daumer M, Scholz M, et al. Sensitivity and specificity of intrapartum computerised FIGO criteria for cardiotocography and fetal scalp pH during labour: multicentre, observational study. Br J Obstet Gynaecol. 2008;115:1557-63.

[16] Scholz C, Koch F, Kost B, Rogenhofer N, Kainer F, Janni W, et al. Teaching obstetrics after the changes in licensing for physicians in Germany. Geburtsh Frauenheilk. 2007;67:1091-6.

[17] Sweha A, Hacker TW, Nuovo J. Interpretation of the electronic fetal heart rate during labor. Am Fam Phys. 1999;59:2487-500.

[18] Weiss PM, Koller CA, Hess LW, Wasser T. How do medical student self-assessments compare with their final clerkship grades? Med Teach. 2005;27:445-9.

The authors stated that there are no conflicts of interest regarding the publication of this article.

Received March 13, 2012. Revised April 28, 2012. Accepted May 11, 2012. Previously published online July 13, 2012. 